\title{
Conductor-based Millimeter Wave Microstrip Patch Antenna for Gain Enhancement
}

\author{
Khadidja Belabbas, Djamel Khedrouche, and Abdesselam Hocini \\ Laboratoire d'Analyse des Signaux et Systèmes, Department of Electronics, \\ Mohamed Boudiaf University of M'sila, BP. 166, Route Ichebilia, M'sila, 28000 Algeria
}

https://doi.org/10.26636/jtit.2021.148320

\begin{abstract}
In this paper, a small $(20 \times 20 \times 2.4 \mathrm{~mm})$ loaded microstrip patch antenna (MPA) with an asymmetric artificial magnetic conductor (AMC) as a ground plane is designed for millimeter wave applications. Two AMC structures are proposed; one has the property of a $0^{\circ}$ reflection phase around $28.4 \mathrm{GHz}$, with a symmetric geometry, which makes the reflection phase insensitive to variations in both polarization and incident angle. This symmetric AMC structure ensures angular stability which is considered as a major requirement when periodic structures are used as antenna ground planes. The other structure is characterized by an asymmetric geometry and shows an interesting behavior around $28.6 \mathrm{GHz}$, where a discontinuity in the reflection phase appeared due to the fact that surface impedance nature changed from purely capacitive to purely inductive. This paper studies the effects of the two proposed AMC structures on the performance of MPAs, by using an array of $8 \times 8$ unit cell elements as an artificial ground plane. Simulation results show that an MPA with a symmetric AMC ground plane offers better impedance matching and a wider bandwidth. Compared with conventional MPAs, gain is enhanced and directivity is improved as well. As far as an MPA with an asymmetric AMC ground plane is concerned, its performance in terms of gain and directivity is higher than that of the conventional solution.
\end{abstract}

Keywords-artificial magnetic conductor, gain enhancement, microstrip patch antenna, millimeter wave, reflection phase.

\section{Introduction}

Millimeter wave frequency bands are receiving much attention today in modern wireless communication systems [1], such as $5 \mathrm{G}$. This part of spectrum covers frequencies from 30 to $300 \mathrm{GHz}$, which corresponds to wavelengths from 1 to $10 \mathrm{~mm}$. The use of this band for the design of a data transmission system offers a number of advantages, for example high throughput and ability of designing a radio link with high directivity [2]. However, communications at frequencies of approximately $60 \mathrm{GHz}$ suffer from high propagation losses due to RF energy absorption by oxygen in the atmosphere [3].
The microstrip patch antenna has been an attractive choice in mobile and wireless communication, because of its low profile, compact design, low cost, easy fabrication and integration with devices. Microstrip patch antennas are also a crucial part of MIMO array aerial systems [4], [5] widely used in $5 \mathrm{G}$ communication applications. Due to such disadvantages as narrow bandwidth, low efficiency and surface wave losses, improved types with big, periodic structures operating at microwave or millimeter wave resonance frequencies are commonly used. These structures are called electromagnetic band-gap (EBG) i.e. are a class of periodic dielectric, metallic, or composite materials that when introduced to an electromagnetic wave, offer a great performance improvement.

A mushroom type EBG has the propriety of a high impedance surface (HIS) and is characterized by in-phase reflections at the resonance frequency [4]-[6]. A uniplanar EBG is a periodic structure with the property of in-phase reflections, just as in the case of the mushroom type EBG, but with no via connection to the ground plane. The absence of via makes its integration with millimeter and microwave circuits easier [7]. The in-phase reflection could be achieved by using the perfect magnetic conductor (PMC) which does not exist in nature. Hopefully, similar characteristics may be achieved by an artificial magnetic conductor (AMC) which is a solution allowing to overcome numerous disadvantages of microstrip antennas operating within the microwave range. It is used as the ground plane to enhance radiation performance, such as uni-directional radiation patterns and the antenna's peak gain [8]-[12]. When the AMC is printed in the same plane as the microstrip antenna, it improves its scattering performance by reducing the radar cross section [13]-[16]. This technique is often used for developing high isolated MIMO antennas [17]. The AMC is also used as a ground plane in textile-based antennas for wearables [18]-[20], in order to suppress back radiation. In [21], a planar circular cross AMC-based ultra-wideband (UWB) antenna is used to enhance the radiation pattern and 
gain. The microstrip patch antenna based on an asymmetric AMC ground plane is also studied in [22], where it is used as a reflection plane to achieve both good bandwidth and a low level of cross-polarization.

In this paper, a simple miniature design of AMC unit cells resonating in the millimeter wave band is presented. The presented unit cell and antenna designs are very simple, making them easy to fabricate and integrate in millimeter wave applications. The presented design has a wide bandwidth, which makes this aerial suitable for broadband millimeter wave antenna applications, and by using an $8 \times 8$ array of unit cells underneath of the conventional microstrip antenna, gain and the directivity are clearly improved as well.

The paper is organized as follows. First, the design and the characteristics of the proposed symmetric and asymmetric AMC are presented in Section 2, where we also analyze their reflection phase and surface impedance properties. In Section 3, the performance of the antenna over an asymmetric AMC ground plane is studied and compared with an MPA with a symmetric AMC ground plane, and with a conventional MPA. Finally, conclusions are presented in Section 4.

\section{Proposed Asymmetric AMC Design}

The AMC material is characterized by two important properties, namely high impedance for transverse electric (TE) and transverse magnetic (TM) wave polarization, and for all propagation directions. Its other peculiar property is the fact that it reflects the impinging waves with a zero-phase shift. It is called AMC, because its tangential magnetic field is zero at the surface.

To study these properties further, we designed a 2D planer asymmetric AMC unit cell. According to [4], the lumped element model describes the AMC structure as an LC resonant circuit. Inductance $L$ and capacitance $C$ are determined by the unit cell geometry and its resonance behavior is used to explain the properties of AMC, such as surface impedance and reflection phase, which are defined as [23]:

$$
\begin{gathered}
Z_{s}=\frac{\mathrm{j} \omega L}{1-\omega^{2} L C}, \\
\omega_{0}=\frac{1}{\sqrt{L C}}, \\
\text { Phase }=\operatorname{Im}\left[\ln \frac{Z_{s}-\eta}{Z_{s}+\eta}\right],
\end{gathered}
$$

where $Z_{s}$ is the impedance surface, $\omega$ is the angular frequency, and $\eta$ is the impedance of free space.

The geometry of the asymmetric unit cell consists of a square patch with two slots with the same width $w=0.1 \mathrm{~mm}$. The slotted patch is placed above a ground FR4 epoxy substrate with dielectric constant $\varepsilon_{r}=4.4$ and

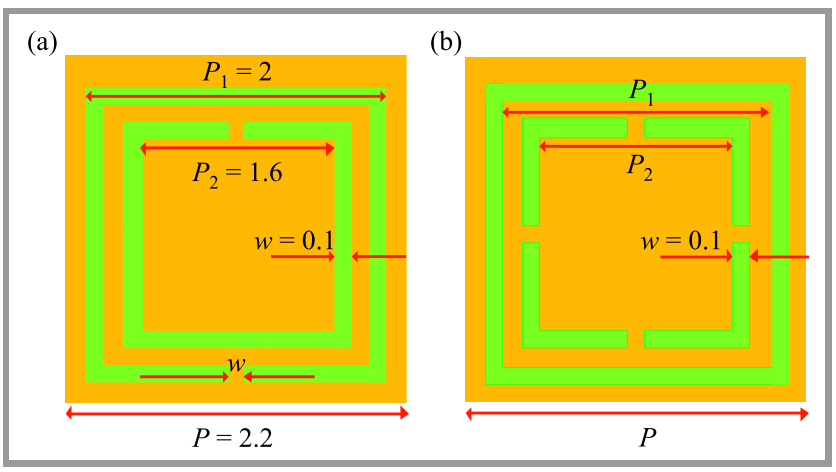

Fig. 1. Top view of the unit cell: (a) asymmetric and (b) symmetric designs.

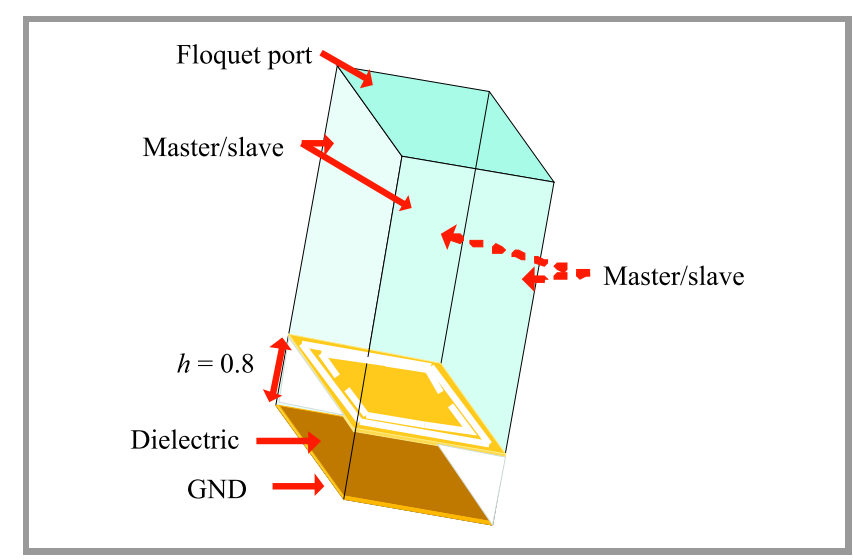

Fig. 2. Simulation model of the unit cell.

$h=0.8 \mathrm{~mm}$. The period of the unit cell is $p$ and the remaining dimensions (in $\mathrm{mm}$ ) are shown in Fig. 1a. HFSS software was used to simulate the characteristics of the unit cell. Figure 2 shows the unit cell simulation, the master/slave boundaries are defined on the side walls of the air box, and the Floquet port is located on its top face to model an infinite periodic structure. The Floquet port allows to conduct research on the incident plane wave for different incident and polarizations angles.

\subsection{Reflection Magnitude}

Figure 3 shows the magnitude of the reflection coefficient when the polarization angle of the incident wave $\varphi=0$ and for different values of incident angle $\theta$. Figure $3 \mathrm{a}$ shows that the asymmetric AMC structure is fully reflective at two resonance frequencies: $25.41-25.62 \mathrm{GHz}$ and 36.34-37.92 GHz. A slight variation in the resonant frequencies is observed for different $\theta$ angles, which means that the asymmetric structure is insensible to variations in the incident oblique angle. We observe also an attenuation in the reflection magnitude at the resonance points. For the lowest resonance frequency, attenuation reaches $-12.88 \mathrm{~dB}$ and $-4.09 \mathrm{~dB}$ for the highest resonances at $\theta=60^{\circ}$.

As far as the symmetric AMC structure is concerned, there is only one resonance frequency and when the incident angle increases, the resonance frequency also increases slightly from $28.6 \mathrm{GHz}$ for normal incidence to $29.1 \mathrm{GHz}$ 
for $\theta=60^{\circ}$ as shown in Fig. 3b. The attenuation in the reflection magnitude is observed and reaches $-3.61 \mathrm{~dB}$ for $\theta=60^{\circ}$, which is less than for the asymmetric AMC.

The magnitude of the reflection coefficient for a TM polarized wave is plotted in Fig. 4 for different $\theta$ values. There are two resonance frequencies for an asymmetric AMC structure, and one for the symmetric version. The resonance point slightly varies with changes in $\theta$. The lowest resonance points are $19.6,18.24$, and $19.13 \mathrm{GHz}$, and the highest ones are $36.33 \mathrm{GHz}, 36.88 \mathrm{GHz}$, and $36.64 \mathrm{GHz}$ for $0^{\circ}, 30^{\circ}$, and $60^{\circ}$, respectively. Note that unlike for the symmetrical AMC structure, the resonance frequencies for TE polarized waves are not identical with those for TM polarized waves, due to the asymmetric geometry of the proposed unit cell. For the sake of clarity, we have only plotted the reflection magnitude of the proposed asymmetric AMC unit cell for $\varphi=0^{\circ}$, because the results for $90^{\circ}$ of the TE polarized wave are equivalent to those of the TM polarized angle for $0^{\circ}$.

\subsection{Reflection Phase}

Here, the reflection phase of the proposed AMC structure for the two polarization states of the impinging plane wave is presented. Figure 5 shows the TE polarization wave and, according to the criterion of the reflection phase being within the range of $-90^{\circ}$ to $+90^{\circ}$, two bands shown in the asymmetric AMC structure. The first is centered on $25.4 \mathrm{GHz}$, and the other on $37.9 \mathrm{GHz}$ unlike in the symmetric geometry, where one band appears around $28 \mathrm{GHz}$. As shown for both AMC structures, the phase changes are insignificant, which means that the proposed structure is not sensitive to oblique incident angle variations. The phase discontinuity for the asymmetric AMC is observed between low- and high-resonance frequencies, which is the most interesting behavior and the main difference between the two studied geometries.

In the case of a TM polarized incident wave, the reflection phase shows the same pattern but both lower and higher resonant frequency points move toward the lower frequency band, as shown in Fig. 6a, with the first band being around $19.6 \mathrm{GHz}$, and the other around $36.3 \mathrm{GHz}$. The point of discontinuity is also shifted downward and centered on $25 \mathrm{GHz}$. One may notice that the phase reflection of the asymmetric AMC is insensitive to the incident oblique angle. The same behavior is observed for symmetric AMC structures, as illustrated in Fig. 6b.

When comparing TE and TM polarized incident waves, one may notice that the reflection phase of the asymmetric AMC structure does not exhibit the same behavior for TE and TM. For the symmetric AMC structure, the center of the operating band is the same and the bandwidth becomes wider than that for TE.

\subsection{Impedance Surface of the Unit Cell}

The impedance surface versus frequency and the behavior of impedance around frequencies where the disconti-

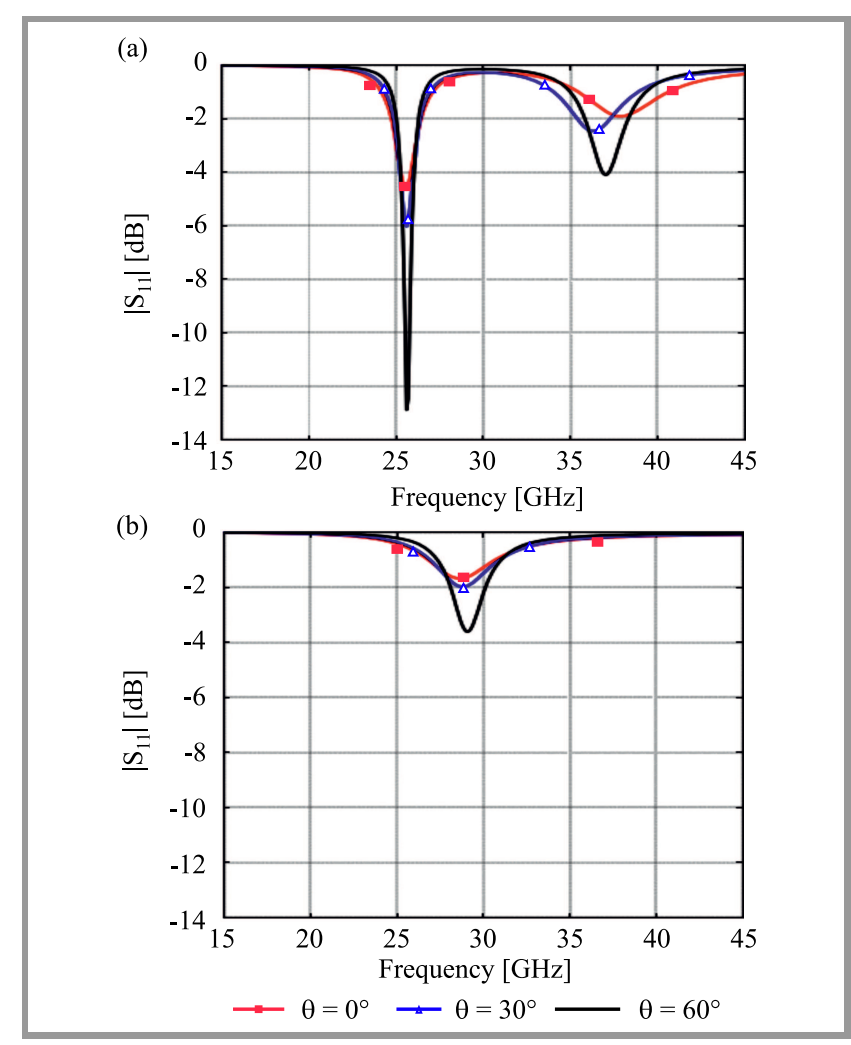

Fig. 3. Reflection magnitude for TE polarization wave, three oblique incident angles and polarization angle $\varphi=0^{\circ}$ : (a) asymmetric AMC and (b) symmetric AMC.

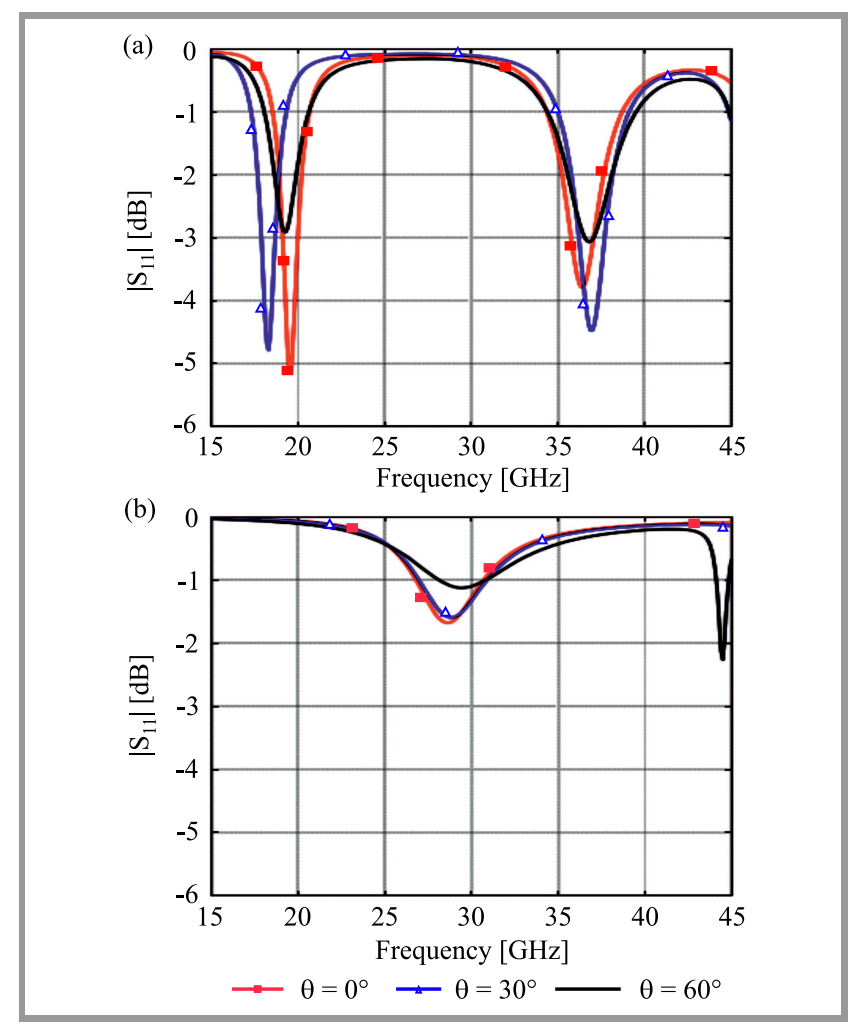

Fig. 4. Reflection magnitude for a TM polarization wave for different oblique incident angles and polarization angle $\varphi=0^{\circ}$ : (a) asymmetric AMC and (b) symmetric AMC. 

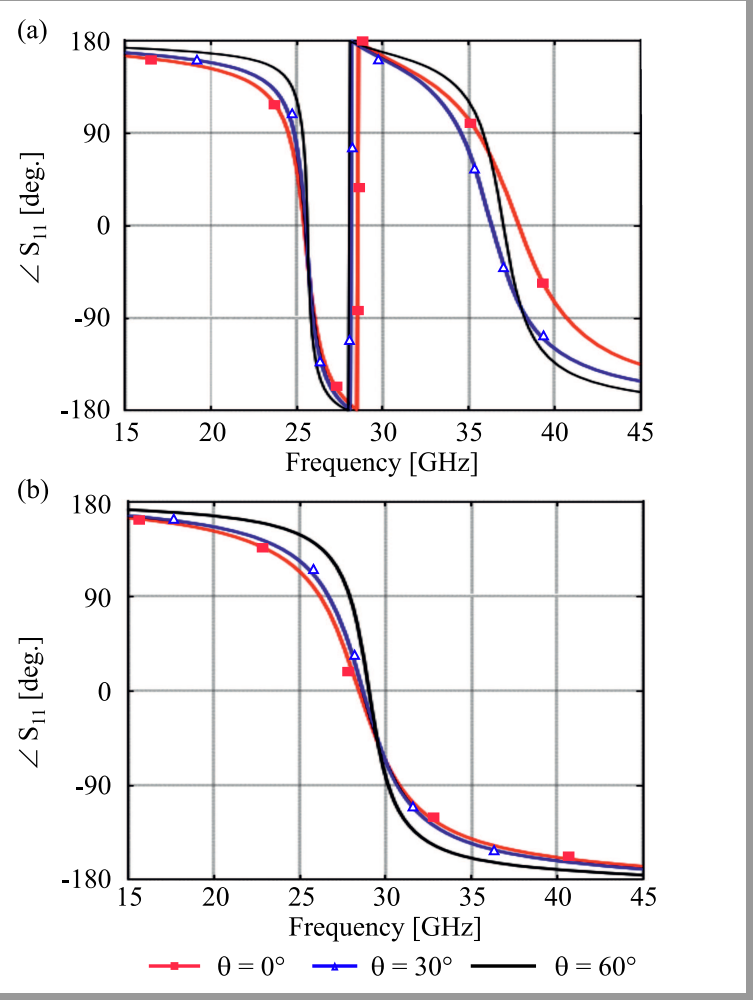

Fig. 5. Reflection phase for a TE polarization wave for different oblique incident angles: (a) asymmetric AMC and (b) symmetric AMC.
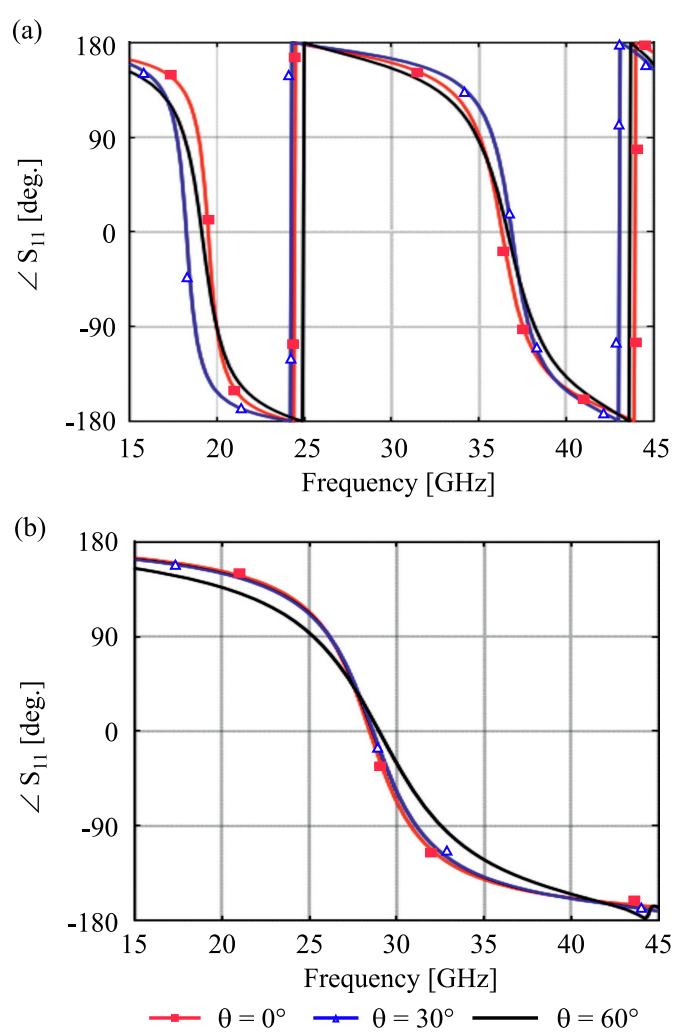

Fig. 6. Reflection phase for a TM polarization wave for different oblique incident angles: (a) asymmetric AMC and (b) symmetric AMC.

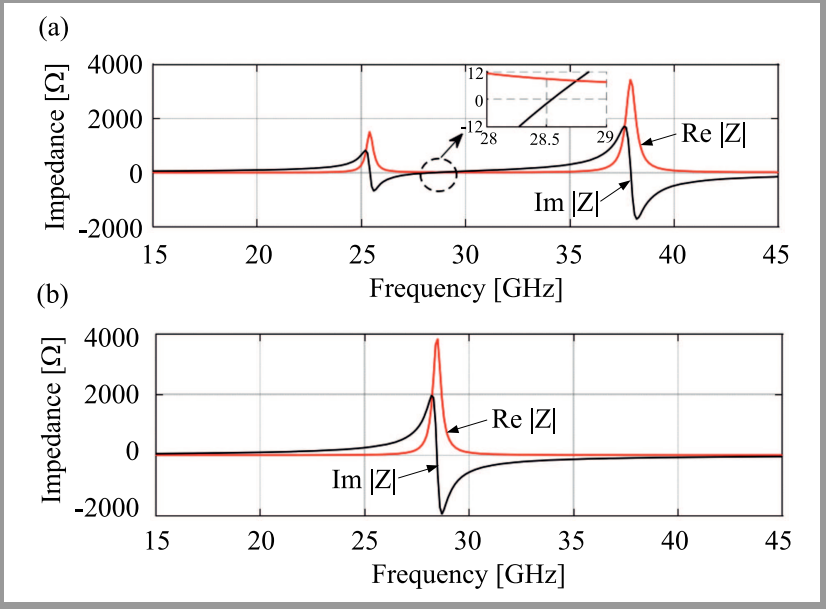

Fig. 7. Impedance of the proposed AMC unit cell for normal incidence and for a TE polarized wave: (a) asymmetric AMC and (b) symmetric AMC.

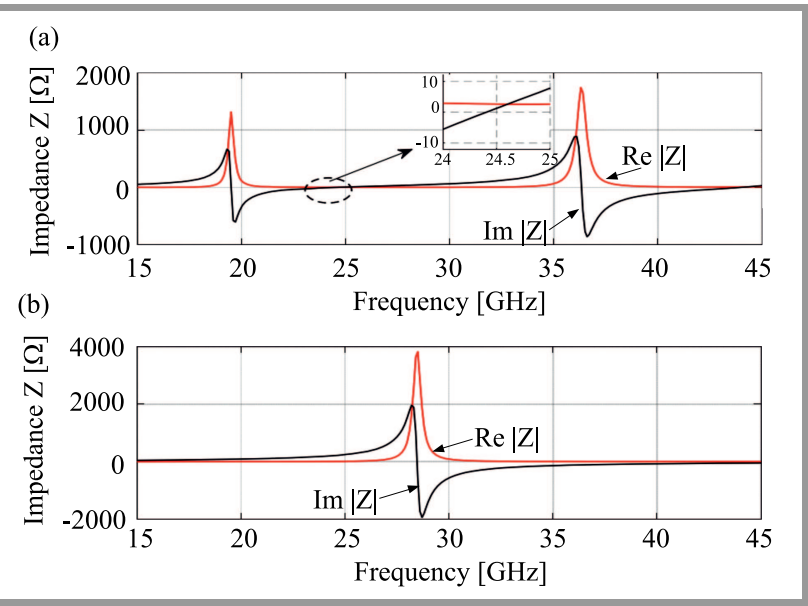

Fig. 8. Impedance surface of the proposed AMC unit cell for normal incidence and for a TM polarized wave: (a) asymmetric $\mathrm{AMC}$ and (b) symmetric AMC types.

nuity of the phase has occurred are shown in Figs. 7-8 both two polarizations. High impedance is observed around $25.4 \mathrm{GHz}$ and $37.9 \mathrm{GHz}$ for the TE polarized wave, and around $19.5 \mathrm{GHz}$ and $36.3 \mathrm{GHz}$ for the TM polarized wave.

These values are identical with the frequencies obtained for the in-phase reflection phase $\theta=0^{\circ}$. At the discontinuity point observed in the phase, the nature of impedance changes from capacitive to inductive, which leads to phase discontinuity. For clarity, a zoomed view around this point of impedance is inserted in Figs. 7 and 8. As we can see, around 28.54 , and $24.45 \mathrm{GHz}$, impedance type changes from capacitive to inductive, for a TE polarized wave and a TM polarized wave, respectively. For the symmetric AMC, the results are in agreement with the reflection phase in terms of resonance frequency points and bandwidth. All resonance points at the $0^{\circ}$ reflection phase are corresponding to those for the maximum value of the real part of impedance. 


\section{Results and Discussion}

The geometry of a conventional microstrip patch antenna with and without the proposed AMC ground plane is shown in Fig. 9. A rectangular microstrip patch antenna of dimensions $W_{p} \times L_{p}$, excited by a $50 \Omega$ microstrip line, is located on a $20 \times 20 \mathrm{~mm}$ FR4 epoxy substrate, with relative permittivity of $\varepsilon_{r}=4.4$ and thickness of $1.6 \mathrm{~mm}$. The same conventional MPA with no dimensional changes is placed directly above this new ground plane, as illustrated in Fig. 9c-d.

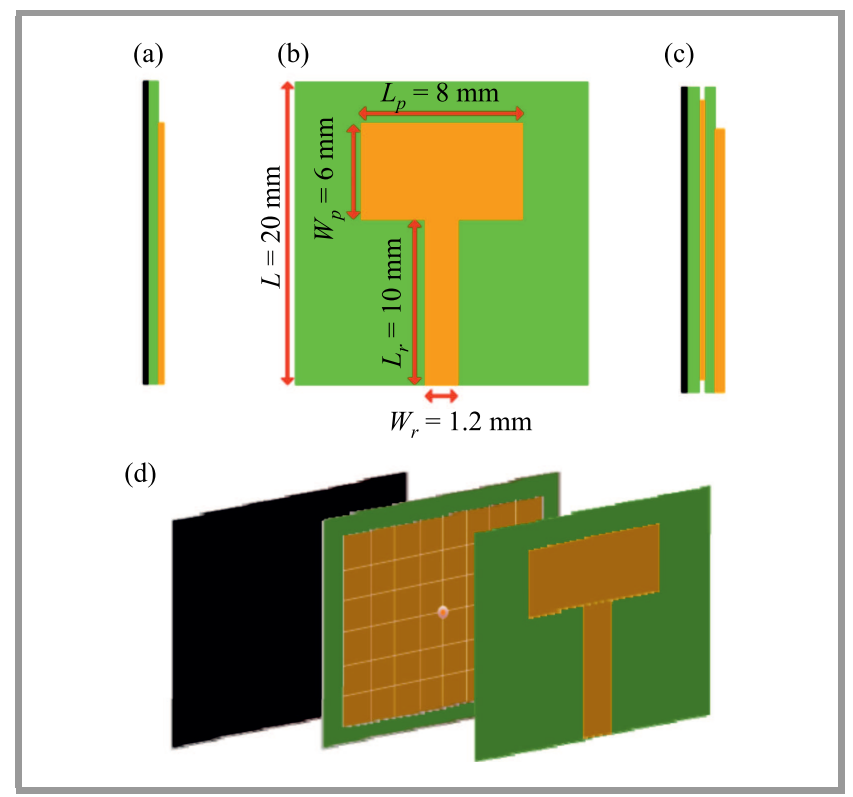

Fig. 9. Proposed microstrip patch antenna design: (a) side view without AMC, (b) front view without AMC, (c) side view with AMC, and (d) position of the dielectric layers.

The return loss of the proposed asymmetric AMC structure ground plane-based MPA is compared, in Fig. 10, with that of the conventional MPA and with that of an MPA based on the well-known symmetric AMC ground plane. The results show good impedance matching, with values of $-46.6 \mathrm{~dB}$ and $-42 \mathrm{~dB}$ being reached for antennas loaded by symmetric and asymmetric AMC ground planes, respectively. This

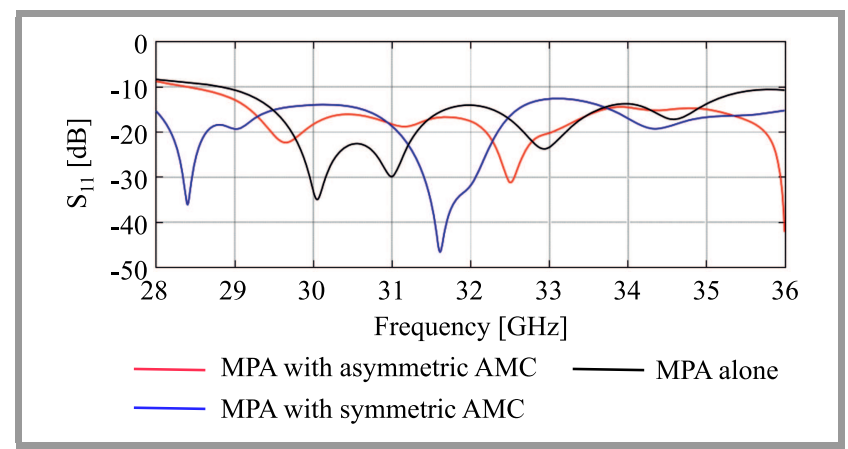

Fig. 10. Return loss for the proposed MPA-based on the AMC structure, compared with that for the conventional MPA and wellknown AMC structure. is due to the in-phase reflection between the image currents created by the radiator patch on the AMC ground plane. The lowest frequency is reduced to $28 \mathrm{GHz}$ and to $28.4 \mathrm{GHz}$ for the antenna loaded by symmetric and asymmetric AMC ground plane, respectively. This is due to the capacitance effect of the AMC structures. However, the higher frequency does not change, which leads to an enhancement in bandwidth. Thus, the antenna with the asymmetric ground plane has an impedance bandwidth of $23.75 \%$, while the bandwidth for the antenna with the symmetric ground plane is $25 \%$, and the bandwidth for the conventional aerial is $22.22 \%$, for $S_{11}<-10 \mathrm{~dB}$.

Gain is depicted, as a function of frequency, in Fig. 11. It reached the maximum around $31.4 \mathrm{GHz}$. This is near to the value where the phase of the asymmetric AMC structure shows discontinuity, and the nature of the impedance surface changes from capacitive to inductive, for a TE polarized wave. A considerable enhancement of gain is achieved, and the peak gain around this point reaches $8.6 \mathrm{dBi}$ for the proposed asymmetric AMC ground plane-based MPA, where the gain of the conventional MPA is $2.8 \mathrm{dBi}$, which means an enhancement of $207.14 \%$. The peak gain for the antenna with the symmetric AMC ground plane is $7 \mathrm{dBi}$ at $31.4 \mathrm{GHz}$, and an enhancement of $148.2 \%$ is achieved. This value of frequency is almost the same as that of the symmetric $\mathrm{AMC}$ unit cell which has the property of a $0^{\circ}$ reflection

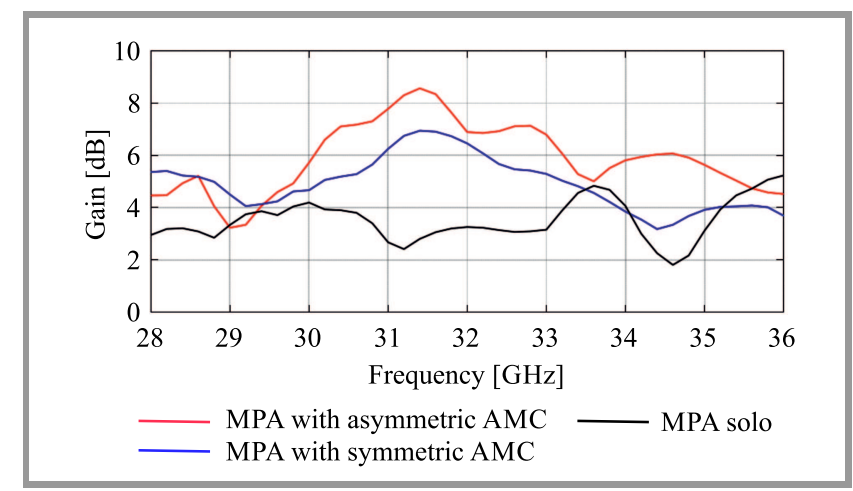

Fig. 11. The peak gain versus frequency for proposed MPA based on asymmetric AMC ground plane compared with that for the conventional MPA and well-known AMC.

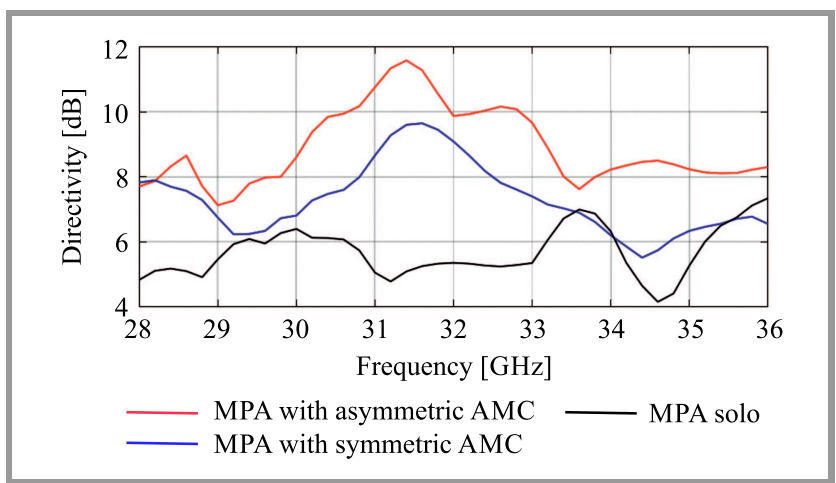

Fig. 12. Peak gain vs. frequency for the proposed MPA based on an asymmetric AMC ground plane compared with results for the conventional MPA and well-known AMC structure. 
(a)

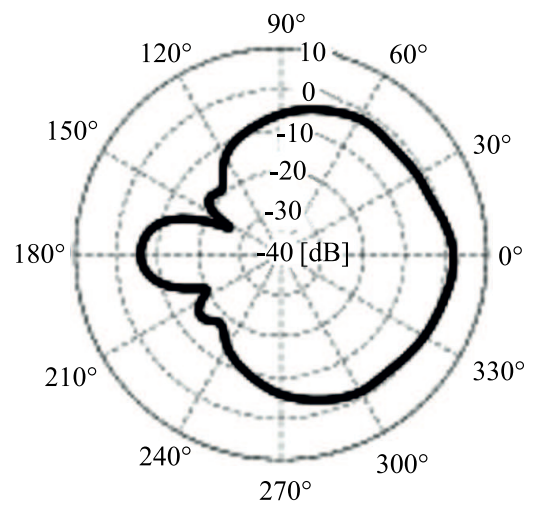

(b)

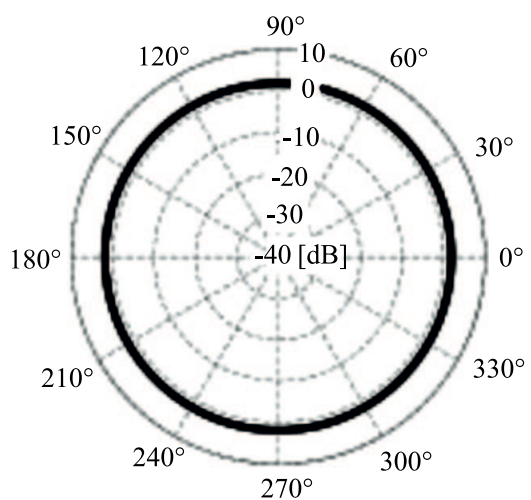

(c)

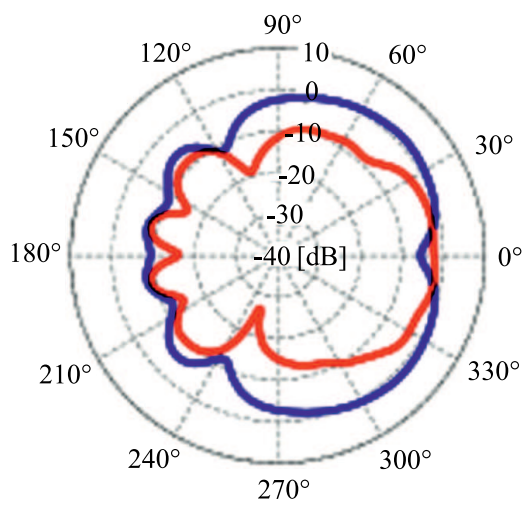

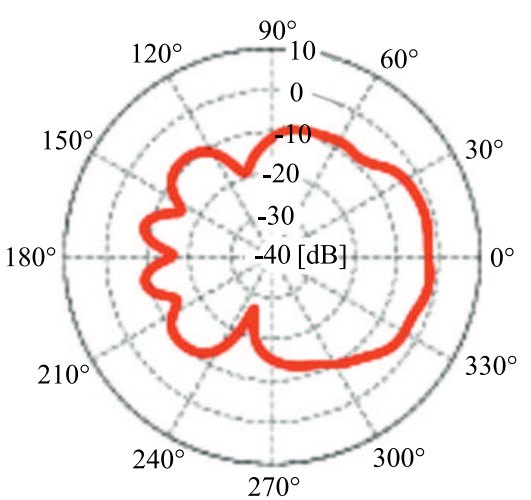
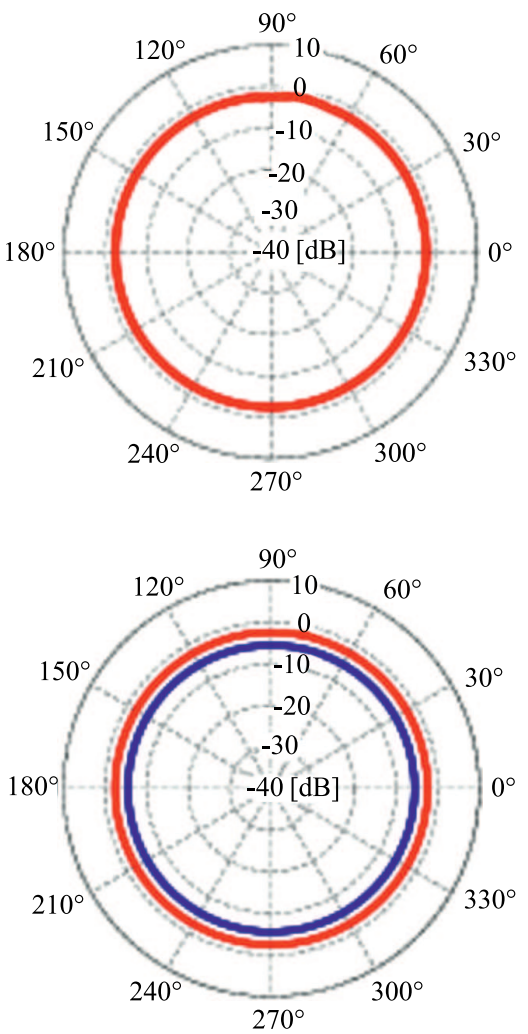

Fig. 13. Simulated radiation pattern for asymmetric type (red line), symmetric version (black line), and conventional antenna (blue line): (a) YOZ plane, (b) XOY plane, and (c) comparison between proposed asymmetric and conventional antennas (the left side is YOZ plane, and the right side is the XOY plane). (See digital version on https://www.itl.waw.pl/en/publications/journal-jtit)

Table 1

Performance comparison of the proposed antenna based on an asymmetric AMC structure and similar designs from recent works

\begin{tabular}{|c|c|c|c|c|c|}
\hline Antenna & $\begin{array}{c}\text { Operating } \\
\text { band [GHz] }\end{array}$ & $\begin{array}{c}\text { Gap } \\
{[\mathrm{mm}]}\end{array}$ & $\begin{array}{c}\text { Gain improved } \\
\text { by [dBi] }\end{array}$ & $\begin{array}{c}\text { Directivity improved } \\
\text { by [dBi] }\end{array}$ & $\begin{array}{c}3 \mathrm{~dB} \\
\text { bandwidth [\%] }\end{array}$ \\
\hline Proposed in [21] & $2.4-11.2$ & 5 & 3.5 & - & 36 \\
\hline From [22] & $24.9-32.1$ & 0.127 & 4 & 4 & 19.94 \\
\hline $\begin{array}{c}\text { MPA with } \\
\text { symmetric AMC }\end{array}$ & $28-36$ & No gap & 4.15 & 6 & 20 \\
\hline $\begin{array}{c}\text { MPA with proposed } \\
\text { asymmetric AMC }\end{array}$ & $28.4-36$ & No gap & 5.8 & 6 \\
\hline
\end{tabular}


phase at $28.46 \mathrm{GHz}$. The difference between the resonance frequency of the AMC structures and the frequency corresponding to the maximum gain is due to the fact that a finite array is used as the ground plane, instead of its infinite variety. In addition, the $3 \mathrm{~dB}$ gain in the bandwidth of the antenna with the symmetric AMC ground plane is $19.94 \%$ (28-34.2 GHz) and 20\% (29.6-36 GHz) for the antenna loaded by the asymmetric AMC ground plane. This corresponds to the operation band of AMC materials. Furthermore, above $35.4 \mathrm{GHz}$, the gain of the MPA alone is higher than that of the MPA loaded by AMC ground planes. Therefore, the AMC structures have a destructive effect on the antenna's radiations, because this range is outside the operation band of the AMC.

The curve of directivity for these antennas is presented in Fig. 12 and is higher. for the proposed aerial, than for the conventional patch antenna. At $31.4 \mathrm{GHz}$, peak directivity for an antenna with the asymmetric AMC ground plane is $11.6 \mathrm{dBi}$, whereas peak directivity for the antenna with the well-known AMC ground plane is $9.6 \mathrm{dBi}$, so that an improvement of $132 \%$ and $92 \%$ is achieved, compared with that for the solo antenna, where the directivity has the maximum value of $5 \mathrm{dBi}$. The enhancement in directivity is caused by the effect of in-phase reflection phase. However, the improvement of directivity for the antenna loaded by the proposed asymmetric AMC ground plane is due to the discontinuity of the reflection phase behavior of the asymmetric AMC, which is higher than that for an MPA based on the well-known AMC ground plane.

For a further understanding of the antennas' performance, the radiation patterns of these antennas are presented in Fig. 13, in both XOY and YOZ planes, at the operating frequency. The omnidirectional radiation pattern is achieved for three antennas in the XOY plane, with a high level of gain for the asymmetric AMC-based microstrip antenna. For the YOZ plane, the radiation pattern for the antenna with the asymmetric AMC exhibits a low level of back lobes and a narrow beam width compared with that for the solo antenna system.

Table 1 shows the performance comparison of the studied antenna and other designs. In terms of peak gain and directivity, the asymmetric AMC ground plane antenna offers better performance. However, the $3 \mathrm{~dB}$ bandwidth is wider in [21], because the antenna is designed for UWB outdoor applications. The gap between the antenna and the AMC ground plane is another important criterion - it equaled, $5 \mathrm{~mm}$ in [21] and $0.127 \mathrm{~mm}$ in [22]. In the proposed design, there is no gap between the antenna and the AMC ground plane, which means that the proposed design is characterized by a lower thickness.

\section{Conclusion}

Conventional microstrip patch antennas with two different AMC ground planes are used to enhance gain and directivity. A symmetric AMC unit cell was designed and placed as the ground plane for a conventional MPA to enhance gain and directivity of the antenna. The unit cell has a simple symmetric geometry, which leads to an angular stability and makes the reflection phase insensitive to polarization and to the incident angle of the incident plane wave. In addition, the $90^{\circ}$ reflection phase bandwidth of $4.8 \mathrm{GHz}(19.2 \%)$ is achieved. The second AMC unit cell has an asymmetric geometry, and it is characterized by the property of discontinuity in the reflection phase, which changes the nature of the impedance surface from capacitive to inductive. The results showed an enhancement by $207.14 \%$ in terms of gain, and by $132 \%$ in terms of directivity. This improvement is higher than that for the symmetric AMC structure which is characterized by the property of a $0^{\circ}$ reflection phase. Both proposed antenna designs with AMC structures achieved better performance in terms of impedance matching, wider bandwidth, and a significant enhancement in gain and directivity, compared to the conventional MPA without an AMC structure.

\section{References}

[1] K. Hamaguchi et al, "Development of millimeter-wave video transmission system-system design and performance for indoor BS signals transmission", in Proc. 2001 Asia-Pacific Microwave Conf., vol. 2, Asia-Pacific, Taipei, Taiwan, 2001, pp. 492-497 (DOI: 10.1109/APMC.2001.985420).

[2] F. K. Schwering, "Millimeter wave antennas", in Proc. IEEE, vol. 80, no. 1, 1992, pp. 92-102 (DOI: 10.1109/5.119569).

[3] A. Elboushi, O. M. Haraz, A. Sebak, and T. Denidni, "A new circularly polarized high gain DRA millimeter-wave antenna", in Proc. IEEE Antennas and Prop. Society Int. Symp., 2010, pp. 1-4 (DOI: 10.1109/APS.2010.5562140).

[4] B. T. P. Madhav, G. J. Devi, P. Lakshman, and T. Anilkumar, "A CPW-fed sigma-shaped MIMO antenna for Ka band and 5G communication applications", J. of Telecommun. and Inf. Technol., vol. 8, no. 4, pp. 97-106, 2018 (DOI: 10.26636/jtit.2018.123717).

[5] S. Agarwal and P. Gupta, "High gain linear $1 \times 4$ x-slotted microstrip patch antenna array for 5G mobile technology", J. of Telecommun. and Inf. Technol., vol. 1, pp. 50-55, 2020 (DOI: 10.26636/jtit.2020.137319).

[6] D. Sievenpiper, L. Zhang, R. F. J. Broas, N. G. Alexopolous, and E. Yablonovitch, "High-impedance electromagnetic surfaces with a forbidden frequency band", IEEE Trans. Microw. Theory Technol., vol. 47, no. 11, pp. 2059-2074, 1999 (DOI: 10.1109/22.798001).

[7] R. Coccioli, F.-R. Yang, K.-P. Ma, and T. Itoh, "Aperture-coupled patch antenna on UC-PBG substrate", IEEE Trans. Microw. Theory Technol., vol. 47, no. 11, pp. 2123-2130, 1999 (DOI: $10.1109 / 22.798008)$.

[8] H. Malekpoor and S. Jam, "Improved radiation performance of low profile printed slot antenna using wideband planar AMC surface", IEEE Trans. Antennas Propag., vol. 64, no. 11, pp. 4626-4638, 2016 (DOI: 10.1109/TAP.2016.2607761).

[9] A. P. Feresidis, G. Goussetis, Shenhong Wang, and J. C. Vardaxoglou, "Artificial magnetic conductor surfaces and their application to low-profile high-gain planar antennas", IEEE Trans. Antennas Propag., vol. 53, no. 1, pp. 209-215, 2005 (DOI: 10.1109/TAP.2004.840528).

[10] M. A. Meriche, H. Attia, A. Messai, and T. A. Denidni, "Gain improvement of a wideband monopole antenna with novel artificial magnetic conductor", in Proc. 17th Int. Symp. on Antenna Technol. and Applied Electromagnetics (ANTEM), 2016, pp. 1-2 (DOI: 10.1109/ANTEM.2016.7550150). 
[11] Y.-W. Zhong, G.-M. Yang, and L.-R. Zhong, "Gain enhancement of bow-tie antenna using fractal wideband artificial magnetic conductor ground", Electron. Lett., vol. 51, no. 4, pp. 315-317, 2015 (DOI: 10.1049/el.2014.4017).

[12] A. Ghosh, V. Kumar, G. Sen, and S. Das, "Gain enhancement of triple-band patch antenna by using triple-band artificial magnetic conductor", IET Microw. Antennas Amp Propag., vol. 12, no. 8, pp. 1400-1406, 2018 (DOI: 10.1049/iet-map.2017.0815).

[13] P. Yao, B. Zhang, J. Duan, and Q. Bai, "A novel low-scattering and wideband monopole antenna based on artificial magnetic conductor", J. Phys. Conf. Serv., vol. 887, no. 1, pp. 12-37, 2017 (DOI: 10.1088/1742-6596/887/1/012037).

[14] Y. Zheng, J. Gao, X. Cao, Z. Yuan, and H. Yang, "Wideband RCS reduction of a microstrip antenna using artificial magnetic conductor structures", IEEE Antennas Wirel. Propag. Lett., vol. 14, pp. 1582-1585, 2015 (DOI: 10.1109/LAWP.2015.2413456).

[15] P. Yao, B. Zhang, and J. Duan, "A broadband artificial magnetic conductor reflecting screen and application in microstrip antenna for radar cross-section reduction", IEEE Antennas Wirel. Propag. Lett., vol. 17, no. 3, pp. 405-409, 2018 (DOI: 10.1109/LAWP.2018.2791662).

[16] D. Sang, Q. Chen, L. Ding, M. Guo, and Y. Fu, "Design of checkerboard AMC structure for wideband RCS reduction", IEEE Trans. Antennas Propag., vol. 67, no. 4, pp. 2604-2612, 2019 (DOI: 10.1109/TAP.2019.2891657).

[17] J. Zhu, S. Li, S. Liao, and Q. Xue, "Wideband Low-Profile Highly Isolated MIMO Antenna With Artificial Magnetic Conductor", IEEE Antennas Wirel. Propag. Lett., vol. 17, no. 3, pp. 458-462, 2018 (DOI: 10.1109/LAWP.2018.2795018).

[18] S. Yan, P. J. Soh, and G. A. E. Vandenbosch, "Low-profile dualband textile antenna with artificial magnetic conductor plane", IEEE Trans. Antennas Propag., vol. 62, no. 12, pp. 6487-6490, 2014 (DOI: 10.1109/TAP.2014.2359194).

[19] S. Lal, K. Subramanya, and R. Abhari, "Miniaturized EBG-backed textile microstrip patch antenna for Bluetooth wearable sensor applications", in Proc. IEEE Int. Symp. on Antennas and Propag. (APSURSI), 2016, pp. 285-286 (DOI: 10.1109/APS.2016.7695851).

[20] S. Zhu and R. Langley, "Dual-band wearable textile antenna on an EBG substrate", IEEE Trans. Antennas Propag., vol. 57, no. 4, pp. 926-935, 2009 (DOI: 10.1109/TAP.2009.2014527).

[21] R. P. Dwivedi, Md. Z. Khan, and U. K. Kommuri, "UWB circular cross slot AMC design for radiation improvement of UWB antenna", AEU-Int. J. Electron. Commun., vol. 117, 2020 (DOI: 10.1016/j.aeue.2020.153092).

[22] M. Xue, W. Wan, Q. Wang, and L. Cao, "Wideband low-profile Kaband microstrip antenna with low cross polarization using asymmetry AMC structure", in Proc. IEEE 69th Electronic Components and Technol. Conf. (ECTC), 2019, pp. 2318-2323 (DOI: 10.1109/ECTC.2019.00319).

[23] D. F. Sievenpiper, "High-Impedance Electromagnetic Surfaces", Ph.D. Thesis, University of California, Los Angeles, California, 1999 [Online]. Available: http://optoelectronics.eecs.berkeley.edu/ ThesisDan.pdf
Khadidja Belabbas is currently pursuing the Ph.D. in Telecommunication and Microwave at Mohamed Boudiaf University, M'sila, Algeria. Her research interests include microstrip patch antennas, EBG-based antennas, and millimeter-wave antennas.

E-mail: khadidja.belabbas@univ-msila.dz

University of Mohamed Boudiaf M'sila

BP.166, Route Ichebilia

M'sila, Algeria

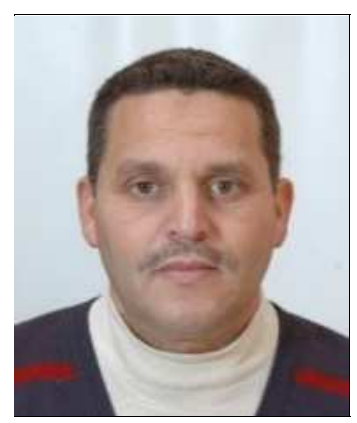

Djamel Khedrouche received his Ph.D. in Electronics Telecommunication in 2009 from Constantine University, Algeria. $\mathrm{He}$ is currently professor in Department of Electronics at University of Mohamed Boudiaf of M'sila, Algeria. His research interests include the modeling and characterization in electromagnetic and microwave devices. Recently, his research concerns microstrip antennas, UWB antennas and realizing advanced functional metamaterial devices.

University of Mohamed Boudiaf M'sila

BP.166, Route Ichebilia

M'sila, Algeria

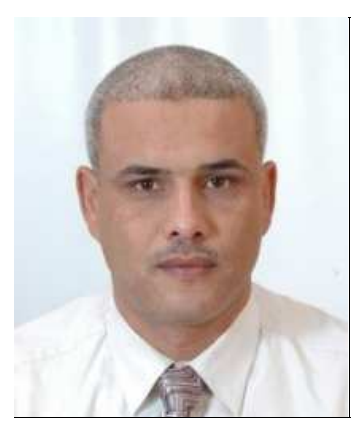

Abdesselam Hocini received his Ph.D. in Electronics Instrumentation in 2008 from Constantine University, Algeria. $\mathrm{He}$ is currently professor in Department of Electronics at University of Mohamed Boudiaf of M'sila, Algeria. His research interests include the design and characterization of photonic devices. In particular, his research concerns sensing, solar cells and realizing advanced functional photonic crystal devices.

University of Mohamed Boudiaf M'sila

BP.166, Route Ichebilia

M'sila, Algeria 